\title{
Numerical Assessment of Airflow and Inhaled Particles Attributes in Obstructed Pulmonary System
}

\author{
A. Lalas, D. Kikidis, K. Votis, D. Tzovaras \\ Information Technologies Institute \\ Centre for Research and Technology - Hellas (CERTH) \\ Thessaloniki, Greece \\ \{lalas, dkikidis, kvotis, dimitrios.tzovaras\}@iti.gr
}

\author{
S. Nousias, A. Lalos, K. Moustakas \\ Department of Electrical and Computer Engineering \\ University of Patras \\ Patra, Greece \\ \{nousias, aris.lalos, moustakas\}@ece.upatras.gr
}

\author{
O. Usmani \\ National Heart and Lung Institute (NHLI) \\ Imperial College London \\ London, United Kingdom \\ o.usmani@imperial.ac.uk
}

\begin{abstract}
Geometry contraction algorithms are introduced in this work to implement the diverse respiratory configurations of lung related diseases associated with airways obstructions. In addition, computational fluid dynamics (CFD) techniques along with fluid particle tracing (FPT) methods are utilized to efficiently evaluate the behavior of the air during the inhalation period, as well as to clarify the features of the inhaled particles in terms of regional deposition. Useful deductions are drawn regarding personalized medication in obstructed conditions.
\end{abstract}

Keywords-computational fluid dynamics; fluid particle tracing; obstructive lung diseases; aerosol deposition; human airways

\section{INTRODUCTION}

Lung diseases, such as asthma and chronic obstructive pulmonary disease (COPD), are life-long chronic inflammatory diseases of the airways affecting over 500 million people worldwide. Several clinical tools have been utilized to assess their severity, supported by ICT infrastructure [1], [2]. Ventilation heterogeneity due to small airways dysfunction is considered an important marker of asthma disease activity, as stated by [3], whereas airborne fine water particles are among the etiological factors that induce asthma attacks in asthmatic children as presented by [4]. In this framework, the basic approach when developing severity assessment tools involves computational fluid dynamics (CFD) simulations [5], [6] accompanied by fluid particle tracing (FPT) analysis of the inhaled ambient particles [7]-[9]. In this way, the airflow characteristics, along with the density and the deposition of the particles on the respiratory airways, are discerned. As of today, various CFD models have been employed to analyze the function of the lungs in terms of inspiration and expiration [10]-[12]. Also, several approaches, based on the data acquired by the CFD analysis, introduce calculations of the particles features [13]-[16].

This work has been funded by the H2020-PHC-2014-2015 Project MyAirCoach (Grant Agreement No. 643607).
Furthermore, authors in [17] introduced a technique to build a multiscale breathing lung model from multidetector computer tomography (MDCT) volumetric data sets acquired at different inflation levels. Additional efforts towards modeling of airway structure (conducting airways), lung function (regional ventilation and boundary conditions), and flow characteristics (modeling of turbulent flow and its effect on particle transport) have been presented by [18]. Multi-scale computational models of the airways have been established by [19] to identify the pathophysiological mechanisms in asthma and COPD. An anatomically-inspired model of the lung acinus featuring a bifurcating tree of alveolated airways with moving walls has been developed [20], [21]. Moreover, the airflow characteristics of human airways between the $8^{\text {th }}$ and $14^{\text {th }}$ generations have been examined [22]. The aforementioned methodologies utilize several turbulence models to evaluate the behavior of the airflow as well as the inhaled particles. However, they do not examine the features of the airflow at the upper generations, i.e. between the $1^{\text {st }}$ and the $5^{\text {th }}$, especially when obstructions are present.

The importance of individual exposure to aerosol particles from a subway microenvironment in the human respiratory tract, along with the dose assessment, in order to provide information for the protection of public health has been described by [23]. As an example, deposition of inhaled particles within airway generations of an adult woman was determined by a stochastic lung deposition model for sleeping, sitting, light and heavy exercise breathing conditions [24]. The most representative approach to deal with the prediction of asthma exacerbations has employed a stochastic lung model to maximize the amount of deposited particles during a severe asthma attack [25]. Other perspectives examine the efficiency of the inhaler techniques adopted for treating asthma [26] as well as the pharmaceutical components in the form of inhaled particles [27], [28]. One of the shortcomings of the existing 
technologies for asthma treatment is the lack of personalization in estimating the required dosimetry of inhaled medicines.

The objective of this work is focused on the accurate modelling of the airways obstructions that relate to pulmonary diseases. In addition, it aspires to provide effective evaluation of the airflow behavior in conjunction with clarification of the inhaled particle properties as an initial step of attaining personalized treatment. To pursue these goals, a geometry processing sequence is developed and utilized for the construction of several important models of the respiratory system. These discrete cases are studied by means of the finite volume method (FVM) and specifically the CFD and FPT algorithms which provide accurate deductions about airflow and deposition fraction upon several parts of the lungs. The data acquired illustrate potential use in personalized medical treatment of lung diseases by providing regional-oriented deposition information.

\section{Structural Modeling, PARAmetrization AND DEFORMATION OF THE LUNG}

Geometry processing approaches are developed to allow the simulation of the effects of the obstructive diseases in the 3D lung structure. This technique enables the study of the impact of any obstructions on the airflow into the lung, by using a CFD simulation. The proposed approaches are executed directly on already available $3 \mathrm{D}$ models of the lung, extracted from CT scans, by using state-of-the-art methods. The surface of a certain part of the mesh is iteratively contracted by executing a Laplacian mesh contraction approach in the direction of the inward normal. Moreover, the lung geometry is segmented into several important components, such as the generations and the outlets, as illustrated in Fig. 1.

\section{A. Lung Geometry contraction in the direction of the Inward Normals}

In order to apply mesh contraction, we employ the Laplacian mesh processing scheme presented in [29]. For the shake of self-completeness, we provide a short overview of the method. A lung 3D model is described by a mesh $(\mathrm{V}, \mathrm{E})$ with vertices $\mathrm{V}$ and edges $\mathrm{E}$. Using cotangent weighting the Laplacian coordinates approximate the curvature-flow inward normal. Thus solving iteratively, the discrete Laplacian equation $L V=0$ we can achieve mesh contraction or reduce the 3D model to an 1D shape. Let $\mathrm{L}$ be the Laplacian operator and $V^{\prime}$ the vertices of the final position, then we have:

$$
\boldsymbol{L}=\left\{\begin{array}{lc}
\omega_{i, j}=\operatorname{cota}_{i, j}+\cot _{i, j} & \text { if }(i, j) \in E \\
\sum_{i, k \in E}^{k}-\omega_{i, k} & i=j \\
\mathbf{0} & \text { otherwise }
\end{array}\right.
$$

where $a, b$ are opposite angles for edge $(i, j)$. Since $L$ is singular, further constrains need to be used in order to ensure a unique solution for $\mathrm{V}^{\prime}$. Thus we focus on solving the equation:

$$
\left[\begin{array}{c}
W_{L} L \\
W_{H}
\end{array}\right] V^{\prime}=\left[\begin{array}{c}
0 \\
W_{H} V
\end{array}\right]
$$

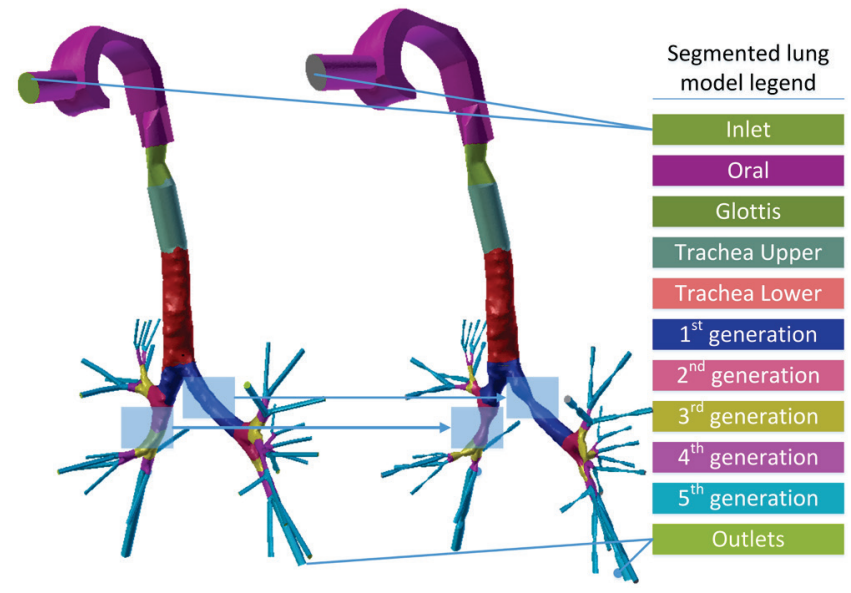

Fig. 1. Patient personalized model of lung geometry up to the $5^{\text {th }}$ generation of the respiratory airways. Normal case in conjunction with the narrowed airways geometry.

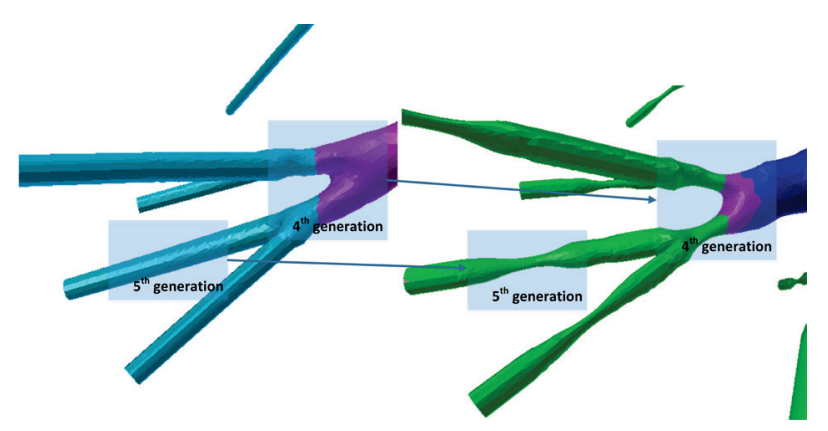

Fig. 2. Geometry of narrowed airways related to the 4th and 5th generation. Narrowing is employed by a percentage of $50 \%$.

where $\mathrm{W}_{\mathrm{L}}$ and $\mathrm{W}_{\mathrm{H}}$ are the diagonal matrices. However, by experimentation one can notice that the solution of (2) does not immediately converge to an $1 \mathrm{D}$ shape and that an iterative scheme has to be employed.

1. Initialize $W_{L}$ and $W_{H}$ in the following manner:

$$
\begin{gathered}
\boldsymbol{W}_{\boldsymbol{L}}=k \sqrt{A} \\
\boldsymbol{W}_{\boldsymbol{H}}=I
\end{gathered}
$$

where $\mathbf{I}$ is a unitary matrix, $\mathrm{k}$ a double constant and $\mathbf{A}$ the average face area of the model.

2. Solve (2) for $\mathbf{V}^{\prime}$

3. Update $W_{L}$ and $W_{H}$ so that

$$
W_{L}^{t+1}=s_{L} \cdot W_{L}^{t}
$$

$$
W_{H, i}^{t+1}=W_{H, i}^{0} \cdot \sqrt{A_{i}^{0} / A_{i}^{t}}
$$




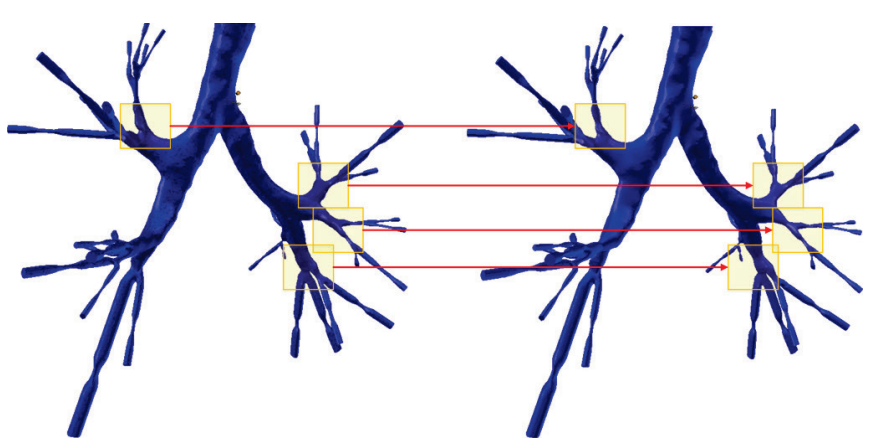

Fig. 3. Magnified perspective of the patient personalized model of lung geometry before and after the narrowing process.

where $s_{L}$ is a user defined double constant, $A_{i}^{0}$ is the initial one-ring area for vertex $\mathrm{i}$ and $A_{i}^{t}$ is the one-ring area for vertex $i$ at iteration $t$.

4. Recompute L.

5. Repeat steps 2 to 4 for a given number of iterations.

Based on the aforementioned scheme we apply mesh contraction at a certain part of the mesh in order to simulate narrowing by using stopping criteria related to the difference between the updated and the initial position. The area under investigation is segmented for more efficient processing and mesh contraction is performed. For this procedure we choose the same attraction weight for $\mathrm{W}_{\mathrm{H}, \mathrm{i}}$ and a different contraction weight $\mathrm{W}_{\mathrm{L}, \mathrm{i}}$ for every node.

However, as the processed part is reconnected with the rest of the mesh, discontinuities and edge effects may appear. In order to cope with this issue, we apply Taubin smoothing [30] at the part of the mesh under process, including the edge points (anchor points) of the stable region of the mesh.

\section{B. Measuring the narrowing percentage}

By inspecting Fig. 2 and Fig. 3, it is made obvious that the airway has been narrowed by a significant percentage. In order to measure how much the airway was narrowed we used a shape diameter function based scheme (SDF) [31]. The SDF produces a scalar value for each face corresponding to the local shape diameter. Thus, by summing up the SDF values of the faces of the processed part of the mesh we derive a characteristic value for the local diameter of the processed segment. The ratio $r$ of the sum of the SDF values before the narrowing process to the sum of the SDF values after the narrowing process corresponds to a metric of the narrowing percentage of the airway. Specifically

$$
r=\frac{\sum_{i=0}^{N} S D F\left(f_{i}^{t}\right)}{\sum_{i=0}^{N} S D F\left(f_{i}^{0}\right)}
$$

where $f_{i}^{t}$ is the face with index $i$ after iteration $t, f_{i}^{0}$ is the initial face with index $\mathrm{i}$ and $\mathrm{N}$ the number of the processed faces. In the current study the processed airways have been narrowed by a percentage of $50 \%$.

\section{Methodology OF LUNG FunCtion ANALYSIS}

The lung geometry is cleaned-up by utilizing several techniques, such as surface reconstruction, surface remeshing, triangulation, and decimation, in order to provide smoothness and facilitate the upcoming procedures of meshing and solving. The meshing is performed by employing the snappyHexMesh algorithm, which is part of an open source platform dedicated to CFD and FPT analyses, called OpenFoam [32]. Our analysis involves hex-dominant cells exhibiting very fine discretization near the walls of the respiratory system. In this way, the interesting properties of turbulence can be resolved, whereas the presence of particles can be accurately clarified. In particular, the domain is discretized into 5569106 cells. In order to effectively deal with the huge computational burden, ARIS, a high performance computer (HPC), was utilized to conduct the related simulations.

\section{A. CFD analysis of pulmonary system}

Reynolds-Averaged Simulation (RAS) is employed to solve the Navier-Stokes equations and perform a steady state CFD analysis of the human pulmonary system. In particular, a kOmegaSST turbulence model is utilized to clarify the properties of the airflow. The study is conducted by means of the FVM technique, especially the SIMPLE algorithm, which is part of the OpenFoam platform. The discretization of the related equations is performed by second order schemes in time and space. In order to enable numerical stability a time step of $5 \times 10^{-4} \mathrm{~s}$ is utilized. A hypothesis of a total pressure drop of $15 \mathrm{~Pa}$ is considered throughout the simulations of inspiratory states. Airflow velocity and pressure are calculated over the computational domain to allow further assessment of inhaled particles behavior.

\section{B. FPT analysis of inhaled particles}

A Lagrangian approach is employed to investigate the motion of particles in fluids by solving a set of ordinary differential equations along their trajectories. The goal of fluid particle tracing is to calculate the change of particle location and the components of the particle velocity. The relevant forces acting on the particle need to be taken into account. Hence, if spherical particles are considered, the differential equations for calculating the particle location and velocity are given by Newtonian second law:

$$
\begin{gathered}
\frac{d x_{P}}{d t}=u_{P}, \\
m_{P} \frac{d u_{P}}{d t}=\sum F_{i}, \\
I_{P} \frac{d \omega_{P}}{d t}=T,
\end{gathered}
$$

where $m_{P}=\rho_{P} d_{P}^{3} \pi / 6$ is the mass of a particle $\left(\rho_{P}\right.$ is the particle density and $d_{P}$ is the particle diameter), $I_{P}=0.1 m_{P} d_{P}^{2}$ is the moment of inertia for a sphere, $F_{i}$ denotes the relevant forces acting on the particle, $u_{P}$ is the linear velocity of a particle, $\omega_{P}$ is the angular velocity of a particle and $T$ is the torque acting on a rotating particle due to the viscous interaction with the fluid. 


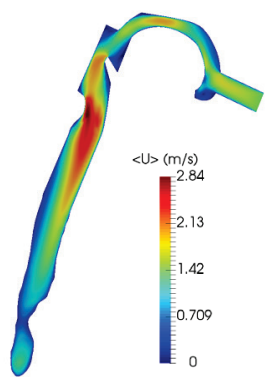

(a)

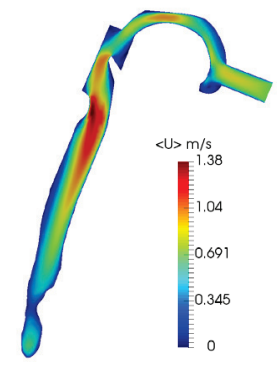

(b)

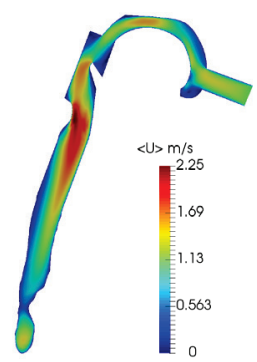

(c)
Fig. 4. Air velocity $(\mathrm{m} / \mathrm{s})$ at the sagital plane cross-section of the upper respiratory tract for several cases: (a) normal respiratory system (b) obstructions in both lungs (c) obstructions in right lung only.
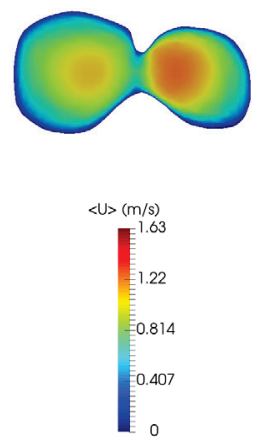

(a)
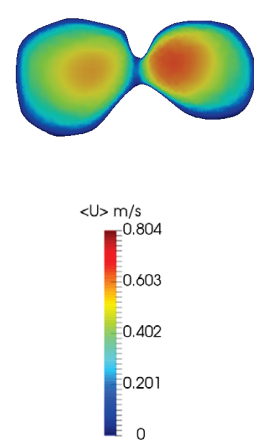

(b)
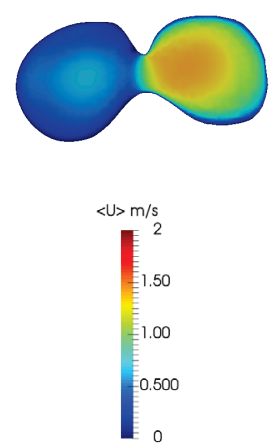

(c)
Fig. 5. Air velocity $(\mathrm{m} / \mathrm{s})$ at the tranverse cross-section at the airways main bifurcation for several cases: (a) normal respiratory system (b) obstructions in both lungs (c) obstructions in right lung only.

For small Reynolds numbers, analytical solutions are available for the different forces applied (Stokes flow). An extension to higher Reynolds numbers is usually obtained by introducing a coefficient $C$ to be multiplied with the force, where $C$ is based on empirical correlations derived from experiments or direct numerical simulations. In most fluidparticle systems, the drag force dominates the particle's motion. An extension to higher particle Reynolds number is based on the introduction of a drag coefficient $C_{D}$, defined from

$$
C_{D}=\frac{F_{D}}{\frac{\rho_{F}}{2}\left(u_{F}-u_{P}\right)^{2} A_{P}},
$$

where $u_{F}$ is the linear velocity of a fluid, $A_{P}=d_{P}^{2} \pi / 4$ is the cross-section of a spherical particle. The drag force can be expressed by:

$$
F_{D}=\frac{3}{4} \frac{\rho_{F} m_{P}}{\rho_{P} d_{P}} C_{D}\left(u_{F}-u_{P}\right)\left|u_{F}-u_{P}\right|
$$

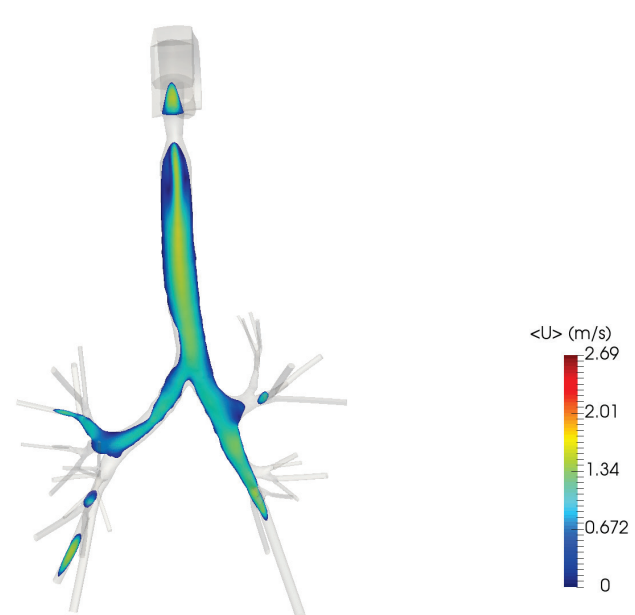

(a)
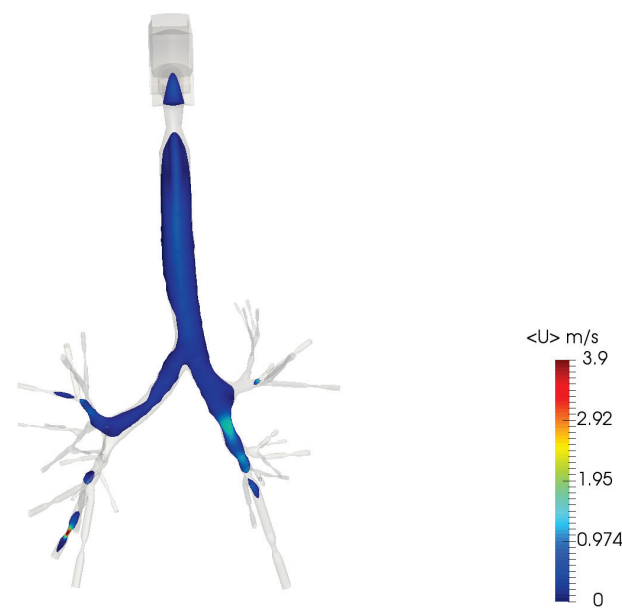

(b)
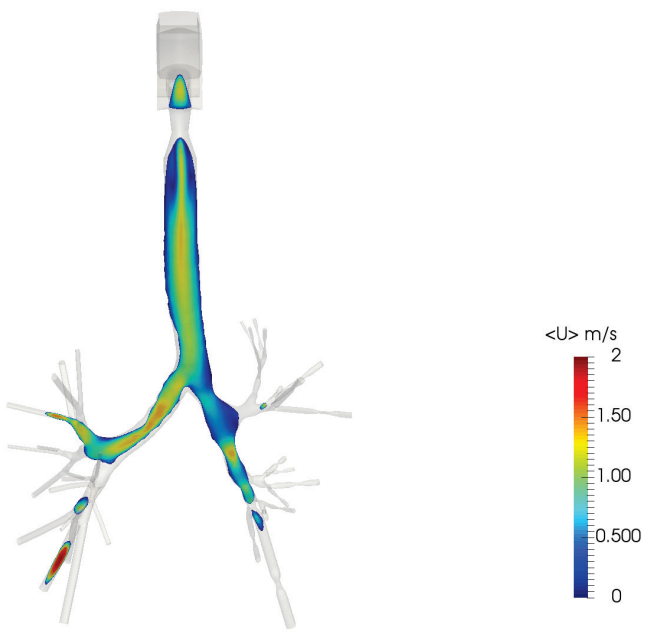

(c)

Fig. 6. Air velocity $(\mathrm{m} / \mathrm{s})$ at the coronal plane cross-section at the trachea and main bronchi for several cases: (a) normal respiratory system (b) obstructions in both lungs (c) obstructions in right lung only. 
The particle Reynolds number that is defined as the ratio of inertial force to friction force:

$$
R e_{P}=\frac{\rho_{F} d_{P}\left|u_{F}-u_{P}\right|}{\mu_{F}},
$$

where $\rho_{F}$ is the fluid density and $\mu_{F}$ is the fluid dynamic viscosity. The aforementioned approach is intended to facilitate particle deposition assessment into the pulmonary system. Moreover, an investigation of the related drug delivery efficiency can be performed to mitigate asthma effects and enable dosimetry conclusions.

A transient FPT analysis is conducted to study the movement of inhaled particles through the respiratory system, as well as the deposition fraction over various areas of interest. Specifically, a one-way coupling between the flow field and the particles is considered, since the steady state field of airflow velocity acquired by the CFD simulation is utilized to calculate the position and velocity of the particles. For the purpose of our simulations, a particle density of $1000.0 \mathrm{~kg} / \mathrm{m}^{3}$ is employed, whereas their diameter is $10 \mu \mathrm{m}$. A total of 54,000 particles are injected through the inlet boundary of the computational domain.

The initial velocity of the particles is considered the same as the inlet velocity of air for the CFD simulations. A stick boundary condition is applied on the wall of the lung geometry as well as on the outlets to efficiently simulate the particle's entrapment on the wall's surface. A transient analysis is performed utilizing the icoUncoupledKinematicParcelFoam solver, which is part of the OpenFoam platform. This algorithm assumes that the particles' effects on turbulence are negligible, thus the flow affects the particles but the particles don't affect the flow. Finally, the position of the particles on the respiratory airways is determined.

\section{NuMERICAL ASSESSMENT OF THE RESPIRATORY SYSTEM}

Several lung models associated to different levels of obstructions have been created using the method described in section I. Each case is determined by the lung generation, after which, obstructions in the form of bottlenecks are observed. Also, an additional case, where only one lung (the right) exhibits obstructions, is modeled. A CFD analysis is performed for each model, whereas the output velocity fields are utilized for the corresponding FPT studies. Then, the particles deposition is calculated for every segment of the lung's model. In this way, deposition fractions are extracted for each generation of the respiratory system, as well as for the left/right sections.

\section{A. CFD Analysis}

The inlet velocity of the air is determined by the simulations, since a total pressure drop of $-15 \mathrm{~Pa}$ is assumed in all cases. For example, the normal lung exhibits an inlet velocity of $1.475 \mathrm{~m} / \mathrm{s}$, while the air velocity when obstructions are observed after the second generation is $0.711 \mathrm{~m} / \mathrm{s}$. Indicative results are presented in Figs. 4-6, where the air velocity distribution cross-sections at the sagital plane, the

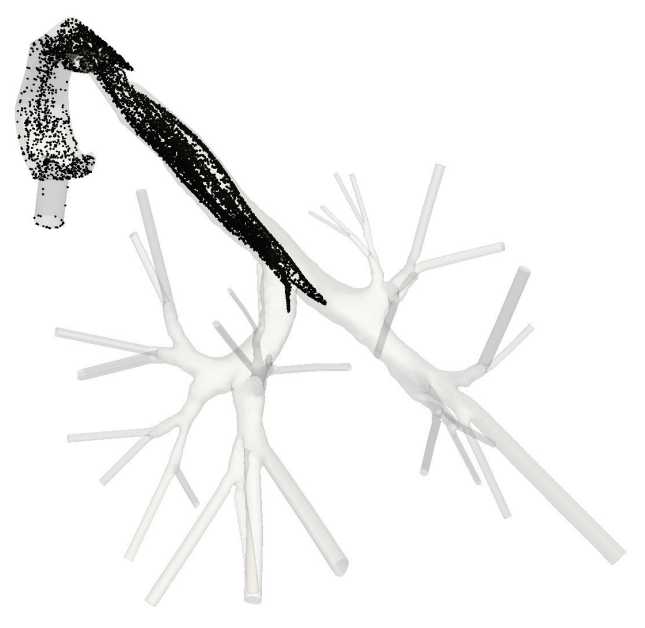

(a)

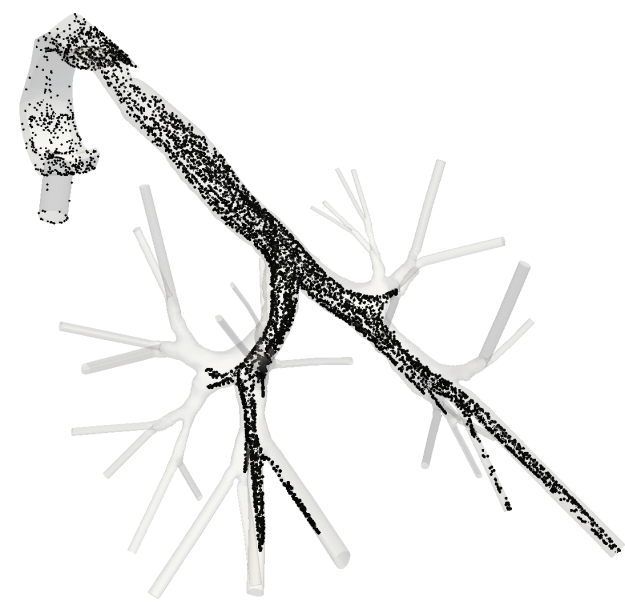

(b)

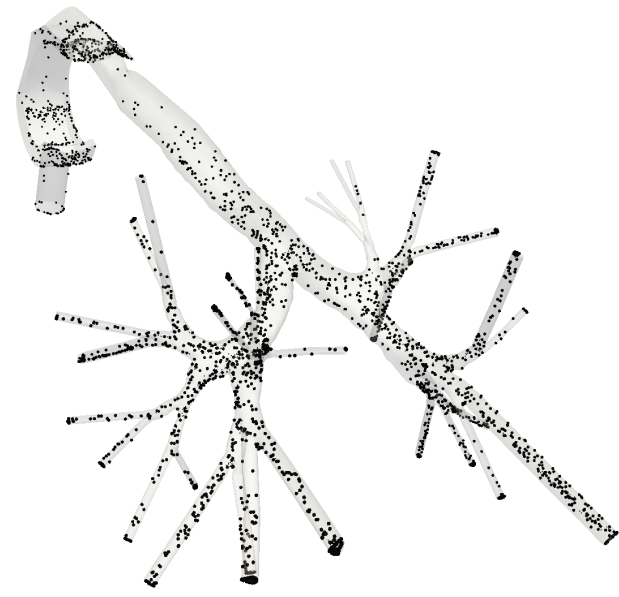

(c)

Fig. 7. Particles position at the region of the normal lung for different time steps during inhalation: (a) $\mathrm{t}=0.2 \mathrm{~s}$ (b) $\mathrm{t}=0.3 \mathrm{~s}$ (c) $\mathrm{t}=0.5 \mathrm{~s}$.

transverse plane, the trachea, and main bronchi, are illustrated. As discerned from Fig. 4, the airflow is reduced at the region of the trachea, when several obstructions occur, thus decreasing 


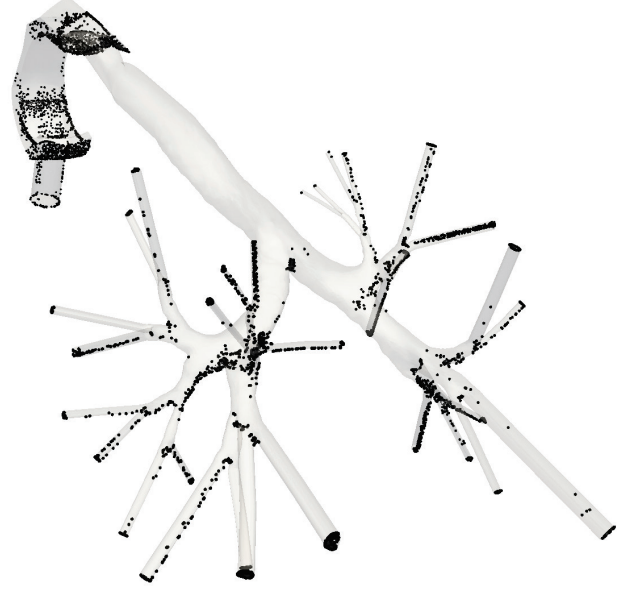

(a)

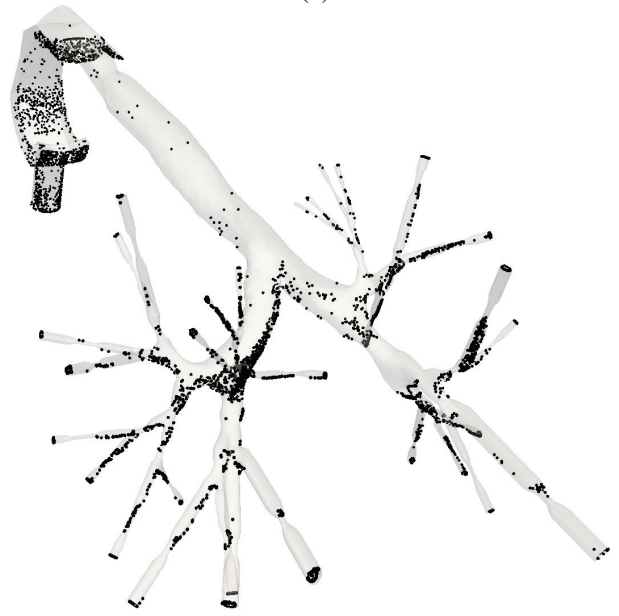

(b)

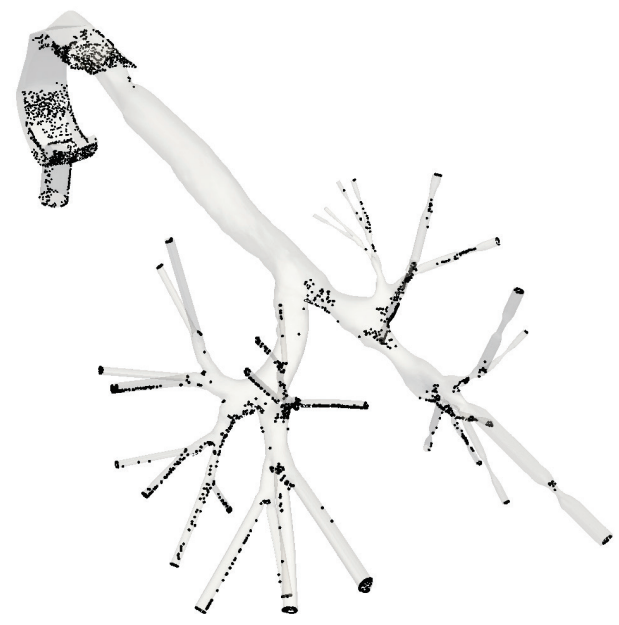

(c)

Fig. 8. Particles position after the end of the inhalation period for different cases: (a) normal respiratory system (b) obstructions in both lungs (c) obstructions in right lung only.

the breathing capability. If only one lung is obstructed the airflow is fairly reduced. Similar deductions can be drawn from
Deposition Fraction (\%)

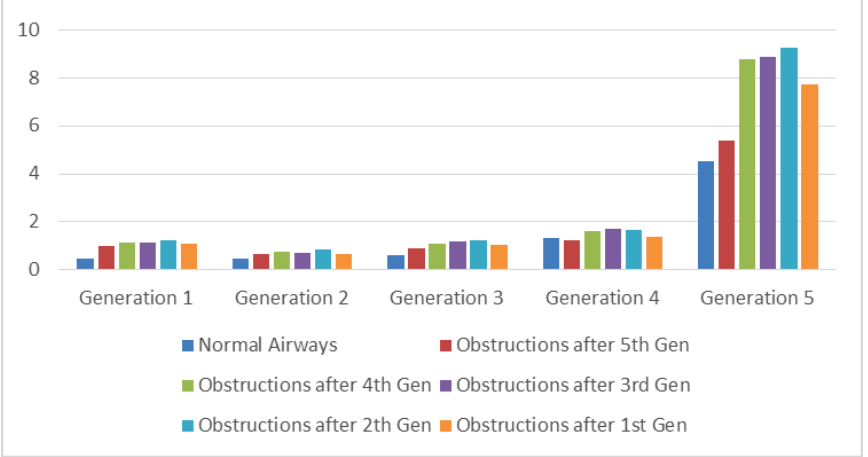

(a)

Deposition Fraction (\%)

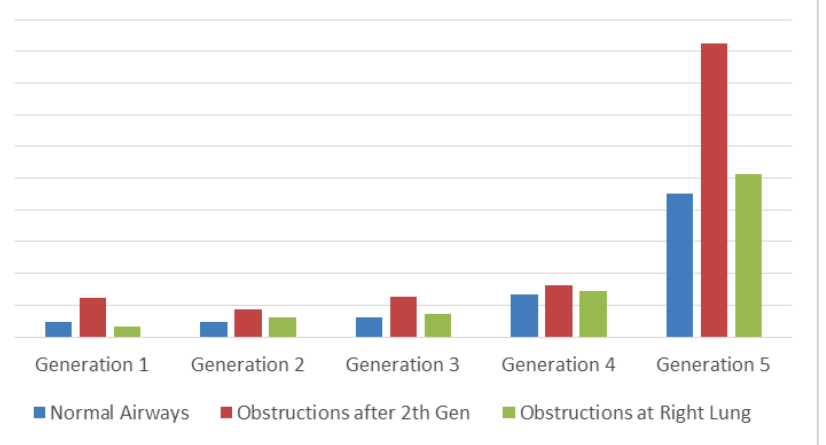

(b)

Fig. 9. Particles deposition fraction over the lung's generations for different cases: (a) Normal vs obstructed airways and (b) normal vs half obstructed airways (right lung is obstructed).

Fig. 5. Also, Fig. 6 clarifies the airflow behavior at the region of the main bronchi. In the case of one obstructed lung, the airflow is driven at the other functional section. This observation denotes that any inhaled particles, ambient or medical oriented, will be guided mostly to the functional part of the respiratory system.

\section{B. FPT Analysis}

A transient analysis of the particles behavior is conducted for each lung model studied in the previous paragraph. The whole inhalation procedure is simulated resulting in a total duration of $2.16 \mathrm{sec}$. Several snapshots of the particles movement into the domain of the normal lung is presented in Fig. 7 for various time steps. Depending on the different conditions and obstruction topologies, the particles will stick on discrete areas of the lung, thus affecting any related diseases. A more comprehensive perspective is attained by the deposition of particles after the inhalation period is completed. Fig. 8 illustrates these depositions for three distinct cases: normal lung, as well as obstructions in both lungs and in the right one only. As observed, when obstructions occur most particles are deposited on their vicinity, in comparison to the normal lung function.

Moreover, particle deposition fraction is calculated for different generations as well as for discrete lung sections. By 
Deposition Fraction (\%)

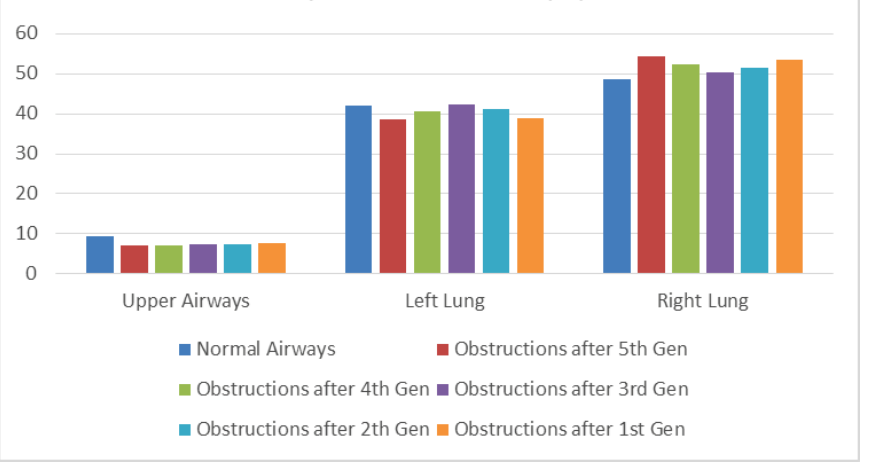

(a)

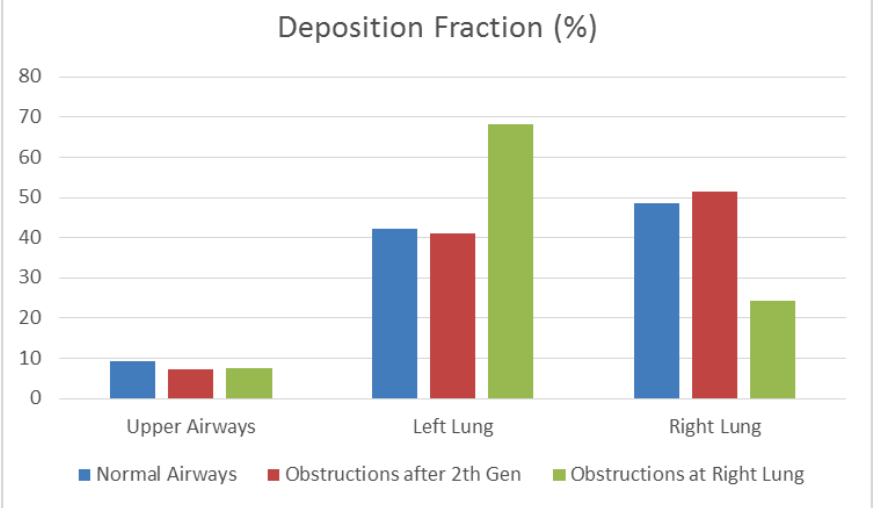

(b)

Fig. 10. Particles deposition fraction over different lung sections: (a) Normal vs obstructed airways and (b) normal vs half obstructed airways (right lung is obstructed).

observing Fig. 9, a maximum deposition on the fifth generation is attained, when obstructions occur all over the respiratory system. Thus, medical oriented particles will be deposited on this area targeting specific treatment. In addition, most particles are located on the right lung, as discerned from Fig. 10, when obstructions occur symmetrically in both lungs. On the other hand, most particles are driven on the left lung, when obstructions are present on the right section only, as expected from the CFD simulations. In this way, any medication will be guided away from the problematic obstructed area. This notice should be taken into account when designing personalized medical treatment of lung diseases.

\section{CONCLUSIONS}

The employment of contraction algorithms to model the different conditions of lung obstructions has been introduced in this paper. Furthermore, accurate CFD and FPT simulations have been utilized to effectively assess the effects of inhaled particles in a regional oriented perspective. Most particles are delivered upon the narrow areas when symmetrical obstructions occur. In addition, partial medication delivery is attained when only one lung is obstructed. This observation could be beneficial when treating lung diseases with inhaled medical substances.

\section{ACKNOWLEDGMENT}

This work was also supported by computational time granted from the Greek Research \& Technology Network (GRNET) in the National HPC facility - ARIS - under project ID 004004.

\section{REFERENCES}

[1] M. Tawhai and P. Hunter, "CT-based geometry analysis and finite element models of the human and ovine bronchial tree," J. Appl. Physiology, pp. 2310-2321, 2004.

[2] P. W. Longest, S. Vinchurkar, and T. Martonen, "Transport and deposition of respiratory aerosols in models of childhood asthma," J. Aerosol Sci., vol. 37, no. 10, pp. 1234-1257, 2006.

[3] F. Thien, "Measuring and imaging small airways dysfunction in asthma.," Asia Pac. Allergy, vol. 3, no. 4, pp. 224-30, Oct. 2013.

[4] K. Kanaya, K. Okamoto, S. Shimbo, and M. Ikeda, "Correlation of light transmittance with asthma attack: Fine water particles as a possible inducing factor of asthma," Environ. Health Prev. Med., vol. 16, no. 1, pp. 44-51, Jan. 2011.

[5] N. Nowak, P. P. Kakade, and A. V Annapragada, "Computational fluid dynamics simulation of airflow and aerosol deposition in human lungs," Ann. Biomed. Eng., vol. 31, no. 4, pp. 374-90, Apr. 2003.

[6] Z. Zhang, C. Kleinstreuer, and C. S. Kim, "Comparison of analytical and CFD models with regard to micron particle deposition in a human 16generation tracheobronchial airway model," J. Aerosol Sci., vol. 40, no. 1, pp. 16-28, 2009.

[7] H. Radhakrishnan and S. Kassinos, "CFD modeling of turbulent flow and particle deposition in human lungs," in 2009 Annual International Conference of the IEEE Engineering in Medicine and Biology Society, 2009, pp. 2867-2870.

[8] A. A. Rostami, "Computational modeling of aerosol deposition in respiratory tract: a review.," Inhal. Toxicol., vol. 21, no. 4, pp. 262-90, Feb. 2009.

[9] J. Löndahl, W. Möller, J. H. Pagels, W. G. Kreyling, E. Swietlicki, and O. Schmid, "Measurement techniques for respiratory tract deposition of airborne nanoparticles: a critical review.," J. Aerosol Med. Pulm. Drug Deliv., vol. 27, no. 4, pp. 229-54, 2014.

[10] V. Srivastav, A. Paul, and A. Jain, "Computational Fluid Dynamics Study of Airflow and Particle Transport in Third to Sixth Generation Human Respiratory Tract," Int. J. Emerg. Multidiscip. Fluid Sci., vol. 3, no. 4, pp. 227-234, Sep. 2011

[11] K. S. Burrowes, T. Doel, and C. Brightling, "Computational modeling of the obstructive lung diseases asthma and COPD.," J. Transl. Med., vol. 12 Suppl 2, no. Suppl 2, p. S5, 2014

[12] D. Wu, M. H. Tawhai, E. A. Hoffman, and C.-L. Lin, "A Numerical Study of Heat and Water Vapor Transfer in MDCT-Based Human Airway Models," Ann. Biomed. Eng., vol. 42, no. 10, pp. 2117-2131, Oct. 2014.

[13] A. H. Alexopoulos, P. Karakosta, and C. Kiparissides, "Particle transfer and deposition using an integrated CFD model of the respiratory system," Comput. Aided Chem. Eng., vol. 28, pp. 211-216, 2010.

[14] A. R. Lambert, P. O'Shaughnessy, M. H. Tawhai, E. a Hoffman, and C.L. Lin, "Regional deposition of particles in an image-based airway model: large-eddy simulation and left-right lung ventilation asymmetry.," Aerosol Sci. Technol., vol. 45, no. 1, pp. 11-25, Jan. 2011.

[15] A. M. Tena, P. Casan, J. Fernández, C. Ferrera, and A. Marcos, "Characterization of particle deposition in a lung model using an individual path," EPJ Web Conf., vol. 45, p. 01079, 2013.

[16] S. Taherian, H. . Rahai, and T. Waddington, "CFD Modeling and Analysis of Pulmonary Airways/Particles Transport and Deposition," in 41st AIAA Fluid Dynamics Conference and Exhibit, 2011, no. June, p. AIAA 2011-3832.

[17] Y. Yin, J. Choi, E. A. Hoffman, M. H. Tawhai, and C. L. Lin, "A multiscale MDCT image-based breathing lung model with time-varying regional ventilation," J. Comput. Phys., vol. 244, pp. 168-192, 2013. 
[18] C. Lin, M. H. Tawhai, and E. A. Hoffman, "Multiscale image-based modeling and simulation of gas flow and particle transport in the human lungs.," Wiley Interdiscip. Rev. Syst. Biol. Med., vol. 5, no. 5, pp. 64355, Sep. 2013.

[19] K. S. Burrowes, J. De Backer, R. Smallwood, P. J. Sterk, I. Gut, R. Wirix-Speetjens, S. Siddiqui, J. Owers-Bradley, J. Wild, D. Maier, and C. Brightling, "Multi-scale computational models of the airways to unravel the pathophysiological mechanisms in asthma and chronic obstructive pulmonary disease (AirPROM).," Interface Focus, vol. 3, p. 20120057, 2013.

[20] P. Hofemeier and J. Sznitman, "Revisiting pulmonary acinar particle transport: convection, sedimentation, diffusion and their interplay," J. Appl. Ph., 2015.

[21] R. Fishler, P. Hofemeier, Y. Etzion, Y. Dubowski, and J. Sznitman, "Particle dynamics and deposition in true-scale pulmonary acinar models.," Sci. Rep., vol. 5, p. 14071, 2015.

[22] B. Sul, A. Wallqwist, M. J. Morris, J. Reifman, and V. Rakesh, "A computational study of the respiratory airflow characteristics in normal and obstructed human airways," Computers in Biology and Medicine, vol. 52, pp. 130-143, June 2014.

[23] V. Martins, M. Cruz Minguillón, T. Moreno, X. Querol, E. de Miguel, M. Capdevila, S. Centelles, and M. Lazaridis, "Deposition of aerosol particles from a subway microenvironment in the human respiratory tract," J. Aerosol Sci., vol. 90, pp. 103-113, 2015.

[24] I. Salma, P. Füri, Z. Németh, I. Balásházy, W. Hofmann, and Á. Farkas, "Lung burden and deposition distribution of inhaled atmospheric urban ultrafine particles as the first step in their health risk assessment,"
Atmos. Environ., vol. 104, pp. 39-49, 2015.

[25] S. Zoltan, "Maximizing the Amount of Deposited Particles During a Severe Asthma Attack Using the Stochastic Lung Model," Acta Medica Marisiensis, vol. 61, no. 3, Jan. 2015.

[26] G. D. Toby, M. R. Capstick, S. Pharm, J. Ian and M. D. Clifton, "Inhaler Technique and Training in People With Chronic Obstructive Pulmonary Disease and Asthma: Effects of Particle Size on Lung Deposition," Expert Rev. Respir. Med., vol. 6, no. 1, pp. 91-103, Feb. 2012.

[27] W. M. C. van Aalderen, J. Grigg, T. W. Guilbert, N. Roche, E. Israel, R. J. Martin, G. Colice, D. S. Postma, E. V. Hillyer, A. Burden, V. Thomas, J. von Ziegenweidt, and D. Price, "Small-particle Inhaled Corticosteroid as First-line or Step-up Controller Therapy in Childhood Asthma," J. Allergy Clin. Immunol. Pract., vol. 3, no. 5, pp. 721-731.e16, 2015.

[28] N. Khajeh-Hosseini-Dalasm and P. W. Longest, "Deposition of particles in the alveolar airways: Inhalation and breath-hold with pharmaceutical aerosols," J. Aerosol Sci., vol. 79, pp. 15-30, 2015.

[29] O. K.-C. Au, C.-L. Tai, H.-K. Chu, D. Cohen-Or, and T.-Y. Lee, "Skeleton extraction by mesh contraction," ACM Trans. Graph., vol. 27, no. 3, p. 1, 2008.

[30] G. Taubin, "Curve and surface smoothing without shrinkage," Proceedings of IEEE International Conference on Computer Vision. pp. 852-857, 1995.

[31] L. Shapira, A. Shamir, and D. Cohen-Or, "Consistent mesh partitioning and skeletonisation using the shape diameter function," Vis. Comput., vol. 24, no. 4, pp. 249-259, Apr. 2008.

[32] OpenFoam v2.4.x. Retrieved from http://openfoam.org 